\title{
Cryo-Electron Tomography Reveals Tegument Density at the Portal Vertex of the HSV-1 Virion
}

\author{
M. F. Schmid*, W. Chiu*, D. Bhella**, F. J. Rixon** \\ *National Center for Macromolecular Imaging, Baylor College of Medicine, Houston, TX, 77030 \\ **MRC Virology Unit, Inst. Of Virology, Glasgow, UK
}

Herpes simplex virus type 1 (HSV-1), the best characterized member of the herpesvirus family, is an important human pathogen. The virion comprises a nominally icosahedral capsid which encloses the genome, a surrounding variable proteinaceous layer called the tegument and an enclosing lipid envelope with glycoprotein spikes. The capsid shell has the form of a $\mathrm{T}=16$ icosahedron and has been studied extensively both as an isolated capsid and as a component of the virion. A 12-fold ring of proteins forms a portal that replaces a penton at one capsid vertex, and, by analogy with tailed bacteriophage, is believed to function in DNA packaging. Here we describe a cryo-electron tomography (cryo-ET) reconstruction of the intact HSV-1 virion that identifies the portal vertex and reveals a previously unsuspected structure that spans the tegument, linking the capsid to the envelope. The position and nature of this structure suggests possible roles in virus assembly or transport.

HSV-1 virions were imaged in JEM2200FS electron microscope using SerialEM and reconstructed by IMOD [1]. Subtomograms of intact enveloped virions were extracted and each capsid was aligned to an icosahedral model derived from single particle reconstruction using a procedure which accounts for the missing wedge [2]. The 12 vertices of each capsid were further distinguished by 3-D classification using multivariate statistical analysis in EMAN [3], requesting 12 classes to allow for the possibility that each of the vertices was unique. Care must be taken when choosing the eigenvectors upon which the classification was based to avoid classification based solely on the missing wedge. Eleven of the 12 classes gave similar 3-D class averages, but one had significant differences in several places: below the capsid floor; at the position occupied by pentons at the other vertices; and outside the capsid, in the tegument region. A difference map between this distinctive vertex and the other vertices was computed, including both positive and negative difference density. This difference map served as a "signature", which was used to reclassify the vertices, and identify a unique "portal" vertex for each particle. Once this special vertex had been chosen for each particle, all particles were oriented to have this vertex coincident. Next, an all-vs.-all comparison of this vertex for all the particles was carried out, with the search constrained to be $0^{\circ}, \pm 72^{\circ}$ and $\pm 144^{\circ}$, between any pair. This would allow alignment of any non-5-fold features at this vertex, as was found for the 6-fold bacteriophage tail fiber [4].

As expected, the resulting asymmetric average of 214 particles (Fig. 1a and b) shows density inside the capsid at a position below the capsid protein floor of the special vertex (Fig. 1b), which corresponds to the previously mapped location of the portal [5]. However, an unexpected feature is the presence of structured density above the portal vertex (Figure 1a and 1b), which we have called the "tegument cylinder" because of its location in the tegument region of the virion. Interestingly, its position is equivalent to that of a bacteriophage tail though its function and chemical identity are not yet known. The tegument cylinder is continuous with the portal and is connected to the surrounding hexons by five densities that resemble "flying buttresses". Overall, this special vertex is characterized by i) a portal/core extending below the floor inside the capsid (Figure 1b), ii) a relatively narrow neck of density connecting the tegument cylinder to the portal. This takes the place 
of a penton, which occupies this location at the other vertices (e.g. the penton at the 6 o'clock position of the capsid, iii) the buttresses connecting to the surrounding triplexes/hexons, and iv) the tegument cylinder density itself, encompassing roughly twice the volume of a hexon and extending all the way out to the envelope. The other eleven vertices show the typical arrangement of tegument density around the penton as previously observed in icosahedral reconstructions from single particles [6].

\section{References}

[1] J.R. Kremer et al., J Struct Biol 116 (1996) 71.

[2] M.F. Schmid et al., J Struct Biol, 161 (2008) 243.

[3] S.J. Ludtke et al., J Struct Biol 128 (1999) 82.

[4] W. Jiang et al., Nature 439 (2006) 612.

[5] J.T. Chang et al., J Virol 81 (2007) 2065.

[6] Z.H. Zhou et al., J Virol 73 (1999) 3210.

[7] This research is supported by NIH grants (5P41RR02250 and 1R01 AI0175208) and Robert Welch Foundation (Q1242). DB and FJR are funded by the UK Medical Research Council.

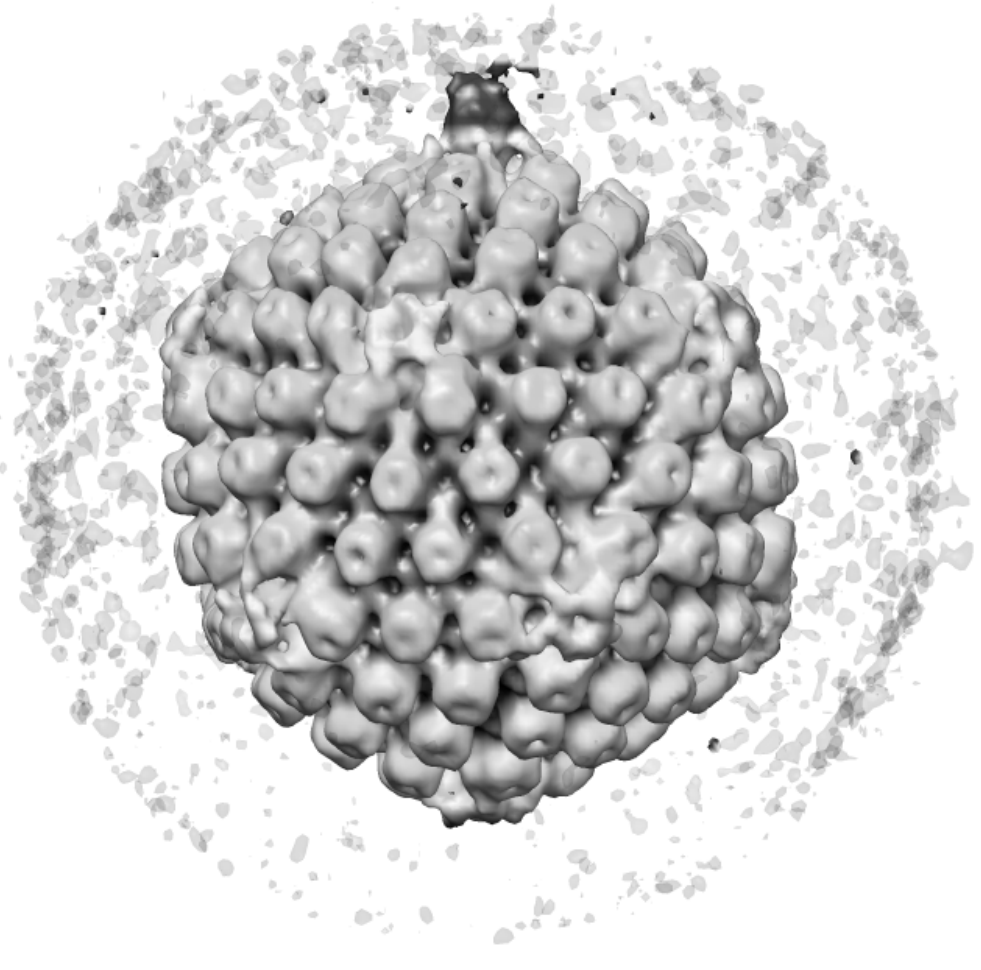

a

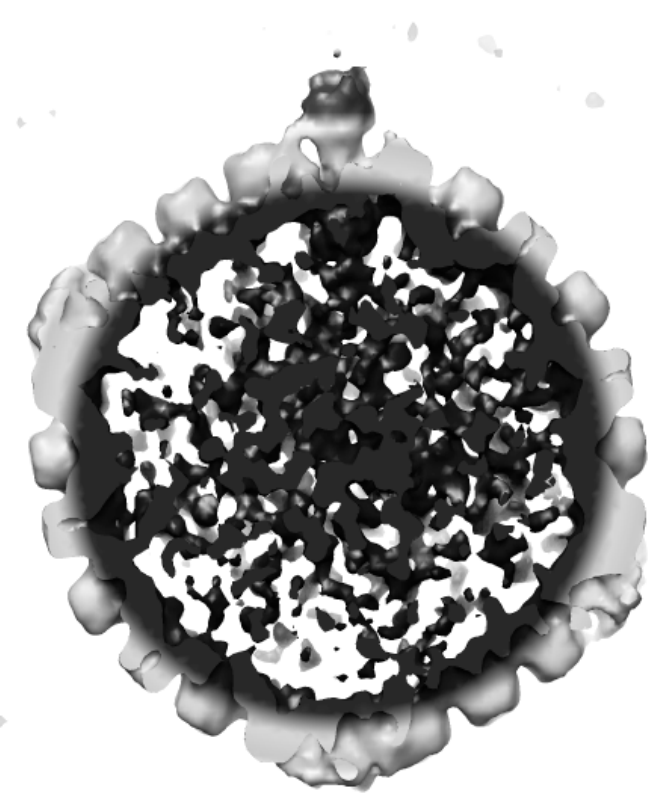

b

Figure 1a. Side view of the subtomogram average of the HSV-1 virion with transparency invoked beyond $760 \AA$ radius to prevent the envelope from obscuring the capsid. The vertex-to-vertex diameter of the capsid is about $1250 \AA$. b. Cutaway view. 